\title{
T cell immunoglobulin and mucin-domain containing-3 in non-small cell lung cancer
}

\author{
Keyi Jia $^{1,2 \#}$, Yayi He ${ }^{1 \#}$, Rafal Dziadziuszko ${ }^{3}$, Sha Zhao ${ }^{1,2}$, Xiaoshen Zhang $^{1,2}$, Juan Deng ${ }^{1,2}$, Hao Wang ${ }^{1,2}$, \\ Fred R. Hirsch ${ }^{4}$, Caicun Zhou ${ }^{1}$, Hui Yu ${ }^{4}$, Liping Zhang ${ }^{5}$ \\ ${ }^{1}$ Department of Medical Oncology, Shanghai Pulmonary Hospital, Tongji University Medical School Cancer Institute, Tongji University School of \\ Medicine, Shanghai 200433, China; ${ }^{2}$ School of Medicine, Tongji University, Shanghai 200433, China; ${ }^{3}$ Department of Oncology and Radiotherapy, Medical \\ University of Gdansk, Gdansk, Poland; ${ }^{4}$ Division of Medical Oncology, Department of Medicine, University of Colorado Anschutz Medical Campus, \\ Aurora 80045, CO, USA; ${ }^{5}$ Department of Pathology, Shanghai Pulmonary Hospital, Tongji University School of Medicine, Shanghai 200433, China \\ Contributions: (I) Conception and design: Y He, FR Hirsch, C Zhou; (II) Administrative support: FR Hirsch, C Zhou; (III) Provision of study \\ materials or patients: R Dziadziuszko; (IV) Collection and assembly of data: K Jia, X Zhang, J Deng, H Wang; (V) Data analysis and interpretation: \\ KJia; (VI) Manuscript writing: All authors; (VII) Final approval of manuscript: All authors. \\ \#These authors contributed equally to this work. \\ Correspondence to: Prof. Caicun Zhou. Department of Medical Oncology, Shanghai Pulmonary Hospital \& Thoracic Cancer Institute, Tongji \\ University School of Medicine, No. 507, Zheng Min Road, Shanghai 200433, China. Email: caicunzhoudr@126.com; Prof. Fred R. Hirsch. Division \\ of Medical Oncology, Department of Medicine, University of Colorado Anschutz Medical Campus, Aurora 80045, CO, USA. \\ Email: Fred.Hirsch@mssm.edu.
}

Background: Immunotherapy has shown promising effect for non-small cell lung cancer (NSCLC) patients. Yet the biomarkers for predicting immunotherapy efficiency are still lacking.

Methods: We tested 139 surgical resected NSCLC primary tumor samples from Medical University of Gdansk, Poland, for T cell immunoglobulin and mucin-domain containing-3 (TIM-3) level by immunohistochemistry (IHC), analyzed the expression of TIM-3 protein on NSCLC tumor cells and tumor infiltrating lymphocytes (TILs).

Results: TIM-3 on TILs was correlated with programmed cell death protein-1 (PD-1) on TILs (correlation coefficient $=0.346, \mathrm{P}<0.001)$ and programmed cell death protein-ligand 1 (PD-L1) on TILs (correlation coefficient $=0.313, \mathrm{P}<0.001$ ), PD-L1 level on tumor cells (correlation coefficient $=0.255, \mathrm{P}=0.002$ ), TIM3 level on tumor cells (correlation coefficient $=0.262, \mathrm{P}=0.002$ ) and TIL percentage (correlation coefficient $=0.172, \mathrm{P}=0.043$ ). TIM-3 level on tumor cells only had correlation with PD-L1 level (correlation coefficient $=0.170, \mathrm{P}=0.045)$. High level of TIM-3 on TILs indicated shorter recurrence-free survival (RFS) and overall survival (OS) (RFS 1.800 years, 95\% CI, 1.230-2.370 vs. 0.870 years, 95\% CI, 0.212-1.528, $\mathrm{P}=0.048$ ) (OS 2.960 years, $95 \%$ CI, 2.268-3.652 vs. 1.080 years, 95\% CI, $0.228-1.932, \mathrm{P}=0.034$ ).

Conclusions: TIM-3 is expressed on NSCLC tumor cells and TILs in all NSCLC pathological type. TIM-3 level on TILs had correlation with PD-1 and PD-L1 level. NSCLC patients with high TIM-3 level on TILs were more likely to have poor prognosis.

Keywords: Non-small cell lung cancer (NSCLC); programmed cell death protein-1 (PD-1); programmed cell death protein-ligand 1 (PD-L1); T cell immunoglobulin and mucin-domain containing-3 (TIM-3); tumor infiltrating lymphocytes (TILs)

Submitted Jul 08, 2019. Accepted for publication Nov 02, 2019.

doi: $10.21037 /$ tlcr.2019.11.17

View this article at: http://dx.doi.org/10.21037/tlcr.2019.11.17 


\section{Introduction}

Lung cancer is increasingly threating the public health all over the world with relatively high morbidity and mortality among all cancers $(1,2)$. Non-small cell lung cancer (NSCLC) is the commonest pathologic type. Conventional chemotherapy has limited effect on advanced stage NSCLC patients. Targeted therapy successfully improves the prognosis of NSCLC patients with driver gene mutations (2-4). However, the population harbors driver gene mutations accounts for only a small part of NSCLC patients (4). Moreover, drug resistance often occurs after a short period of targeted therapy (4).

Recent years, immunotherapy hits the spotlight with its remarkable breakthrough in cancer treatment (5-7). Unfortunately, the mechanism of tumor immunotherapy is not fully understood. Thus, approximately only one quarter of patients could be benefited from programmed cell death protein-1 (PD-1) or programmed death ligand-1 (PD-L1) blockade therapies. What's worse, resistance to immunotherapy remains a main obstacle to extending overall survival (OS), and the mechanisms of drug resistance are still largely unknown. Further studies of immune checkpoints and cancer immune microenvironments are required in order to promote the outcome of immunotherapy. Among these studies, approaches focus on other immune checkpoints are considered of great potential.

$\mathrm{T}$ cell immunoglobulin and mucin-domain containing-3 (TIM-3) belongs to the Ig superfamily (8). TIM-3, as a cell membrane protein marker, was first discovered on Th1 and Tc1 cells which produce IFN- $\gamma$ (8). TIM-3 suppressed T cell functions. The impairment of TIM-3 function could cause autoimmune diseases, such as multiple sclerosis and Crohn's disease $(8,9)$. TIM-3 is often co-expressed with $\mathrm{PD}-1$ and some other inhibitory surface marker in exhausted T cells (10). The frequency of TIM-3(+) CD8(+) $\mathrm{T}$ cells, which inhibiting cytokine production, was elevated in human immunodeficiency virus 1 (HIV-1) infection (11). Recently, TIM-3 was reported to be correlated with antitumor immunity. Using mouse model, Sakuishi et al. found that over half population of CD8 + tumor infiltrating lymphocytes (TILs) co-expressed TIM-3 and PD-1 (12). The co-expression of TIM- 3 and PD- 1 marked the most exhausted CD8(+) T cell phenotype (13). Moreover, it was reported that TIM-3 upregulation in NSCLC patients might induce resistance to therapeutic PD-1 blockade (14). Nowadays, several anti-TIM-3 monoclonal antibodies were currently in clinical trials. TIM-3 blockade therapy was considered of high potential in enhancing anti-PD-1/L1 therapy when combined and may overcome the resistance to anti-PD-1/L1 treatment. This study elucidated the correlation between the expression patterns of TIM-3 and other checkpoints in NSCLC, and their correlation with survival.

\section{Methods}

\section{Patient samples}

We collected 139 surgical resected primary lung cancer specimens from Medical University of Gdansk, Poland (ethical number 15-235), which we mentioned in our published paper (15). Patients had not undergone any kind of treatment before surgery. The surgical histology reports were reviewed and the lymph node and lung cancer stages were categorized by 7 th edition International Association for the Study of Lung Cancer (IASLC) TNM staging system. All participants were competent to provide their consent.

\section{Immunobistochemistry (IHC) by Ventana benchmark XT ${ }^{\circledR}$}

IHC was performed by Ventana benchmark XT ${ }^{\circledR}(15)$. Primary antibodies (TIM-3, D5D5R, 1:500, Cell Signaling) were applied.

\section{Select the IHC cutoff value}

The pathologic tests were completed by two experienced pathologists independently. All samples were reviewed. TIM-3 positive on TILs was confirmed only when there was more than $10 \%$ staining. On tumor cells, the cutoff was rather low $(>5 \%)$ because TIM-3 was rarely found on tumor side. We tested different cutoffs with survival analysis to decide the best cutoff, when the statistical differences of recurrence-free survival (RFS) and OS were maximized simultaneously $(15,16)$.

\section{Evaluation of TILs}

According to previous study, we calculated the number of lymphocytes within each histo spots (17). The fraction of TILs was divided into three level: $<30 \%$ was low, $30-60 \%$ was medium and $>60 \%$ was rather high. Any spot categorized into different level were rechecked by two pathologists together until a consensus was reached (18). 


\section{Data analysis}

The relationship between TIM-3 expression and clinical pathological parameters was evaluated by Chi-square tests. Expression level of TIM-3 and PD-1/L1 were tested by Spearman's correlation tests, in order to analyze the association between checkpoints. We also performed logistic regression to analyze the relationship between TIM-3 and factors including age, gender, smoking history, lung cancer stage, grade, PD-1 and PD-L1 level. The survival distributions of different groups were compared using the Kaplan-Meier method. We also performed univariate and multivariate Cox regression. The hazard ratios (HRs) and their $95 \%$ confidence intervals (95\% CIs) were computed after adjusting by age, gender, smoking status, $\mathrm{T}$ stage, $\mathrm{N}$ stage, $M$ stage, lung cancer stage and grade. The endpoint for RFS was tumor relapse. The endpoint for OS was death from any cause. All $\mathrm{P}$ values were 2-sided, and statistical significance was defined as $\mathrm{P}<0.05$. Statistical analysis was performed using SPSS statistical software package (version 17.0; SPSS, Inc.; Chicago, IL, USA).

\section{Results}

\section{Clinical characteristics of patients}

From April 2010 to August 2011, Medical University of Gdansk, Poland provided us with 139 surgical resected NSCLC specimens along with their clinical data. In these patients, $109(78.4 \%)$ were male and $30(21.6 \%)$ were female. Average age was 64 . Six $(4.3 \%)$ patients had no smoking history. Fifty-eight (41.7\%) patients had stage I NSCLC, 35 (25.2\%) had stage II, 39 (28.1\%) had stage III, and $7(5.0 \%)$ had stage IV. Forty $(28.8 \%)$ patients had adenocarcinoma, and $81(58.3 \%)$ had squamous cell carcinoma (SCC) (Table 1).

\section{Association between TIM-3 and clinical parameters}

In all tested samples, 9 (6.5\%) had positive TIM-3 expression on tumor cells (>5\% staining), 11 (7.9\%) had TIM-3 positive TILs (>10\% staining) (Figure 1). TIM3 positive tumors had higher chance of metastasis, though not statistically significant $(5.3 \%$ vs. $28.6 \%, \mathrm{P}=0.066)$. The distributions of other clinical characteristic, including age, gender, smoking history, stage, pathologic type and grade, were even when grouped with TIM-3 on either side. The negative effect of tumor side TIM-3 on the number of TILs was observed but not significant. However, we did find that
TIM-3 positive tumors were more likely to have TIM-3 positive TILs, and vice versa (Table 2).

\section{Correlation between TIM-3 and PD-1/L1}

We use the Spearman's correlation test to describe the connection between the level of TIM- 3 and other checkpoints. All patients were included in this analysis. The IHC scores was evaluated as grade variables. We found thatTIM-3 level on TILs had broad connections with other checkpoints including PD-1 (correlation coefficient $=0.346, \mathrm{P}<0.001$ ) and PD-L1 (correlation coefficient $=0.313, \mathrm{P}<0.001$ ). Interestingly, TIM-3 level on TILs also had positive relation with PD-L1 level on tumor cells (correlation coefficient $=0.255, \mathrm{P}=0.002$ ), TIM-3 level on tumor cells (correlation coefficient $=0.262, \mathrm{P}=0.002$ ) and TIL percentage (correlation coefficient $=0.172$, $\mathrm{P}=0.043)$. However, TIM-3 level on tumor cells only had correlation with PD-L1 level (correlation coefficient $=0.170, \mathrm{P}=0.045$ ) (Table 3).

\section{Logistic regression for TIM-3 expression}

With the logistic regression model, we performed univariate and multivariate analysis. After adjusting for age, gender, smoking status, pathologic type and tumor stage, the OR for TIM-3 expression on tumor cells was 5.318 (95\% CI, 1.298-21.785; P=0.02) when samples showed PDL1 positive on tumor cells compared with those showed negative on tumor cells (Tables $S 1, S 2$ ).

\section{Association between TIM-3 expression and survival in NSCLC}

The median PFS of 139 patients was 1.720 years, 92 (66.2\%) patients had relapse. Median OS was 2.780 years, 92 (66.2\%) patients reached the end event. For 93 stage I-II patients, $52(55.9 \%)$ had the end event for PFS with a median of 3.090 years, $50(53.8 \%)$ had the end event for OS with a median of 3.280 years. For 46 stage III-IV patients, 40 $(87.0 \%)$ had relapse with a median PFS of 0.660 years, $42(91.3 \%)$ reach the end event of OS with a median of 0.730 years. The cutoff we used for KM test were as aforementioned, $>5 \%$ on tumor cells and $>10 \%$ for TILs. TIM-3 level on tumor cells failed in predicting RFS or OS, although the positive group had slightly shorter survival (RFS 1.760 years, $95 \%$ CI, $1.246-2.274$ vs. 0.760 years, $95 \%$ CI, 0.497-1.023, $\mathrm{P}=0.622$; OS 2.790 years, $95 \%$ CI, $1.970-3.610$ 
Table 1 Characteristics of the 139 patients from Medical University of Gdansk

\begin{tabular}{|c|c|}
\hline Characteristic & Number, n (\%) \\
\hline \multicolumn{2}{|l|}{ Gender } \\
\hline Male & $109(78.4)$ \\
\hline Female & $30(21.6)$ \\
\hline \multicolumn{2}{|l|}{ Age, median } \\
\hline$<70$ & $105(75.5)$ \\
\hline$\geq 70$ & $34(24.5)$ \\
\hline \multicolumn{2}{|l|}{ Smoking status } \\
\hline Non-smoker & $6(4.3)$ \\
\hline Smoker & $133(95.7)$ \\
\hline \multicolumn{2}{|l|}{ Surgery } \\
\hline Wedge & $2(1.4)$ \\
\hline Segmentectomy & $3(2.2)$ \\
\hline Lobectomy & $73(52.5)$ \\
\hline Bilobectomy & $8(5.8)$ \\
\hline Pneumonectomy & $47(33.8)$ \\
\hline Sleeve lobectomy & $6(4.3)$ \\
\hline \multicolumn{2}{|l|}{ T stage } \\
\hline 1 & $25(18.0)$ \\
\hline 2 & $80(57.6)$ \\
\hline 3 & $24(17.3)$ \\
\hline 4 & $10(7.2)$ \\
\hline \multicolumn{2}{|l|}{$\mathrm{N}$ stage } \\
\hline 0 & $75(54.0)$ \\
\hline 1 & $31(22.3)$ \\
\hline 2 & $33(23.7)$ \\
\hline \multicolumn{2}{|l|}{ M stage } \\
\hline 0 & $132(95.0)$ \\
\hline 1 & $7(5.0)$ \\
\hline \multicolumn{2}{|l|}{ Lung cancer staging } \\
\hline I & $58(41.7)$ \\
\hline II & $35(25.2)$ \\
\hline III & $39(28.1)$ \\
\hline IV & $7(5.0)$ \\
\hline \multicolumn{2}{|l|}{ Pathology } \\
\hline SCC & $81(58.3)$ \\
\hline Adenocarcinoma & $40(28.8)$ \\
\hline Large cell carcinoma & $4(2.9)$ \\
\hline
\end{tabular}

Table 1 (continued)
Table 1 (continued)

\begin{tabular}{lc}
\hline Characteristic & Number, $\mathrm{n}(\%)$ \\
\hline NSCLC NOS/mixed & $12(8.6)$ \\
NSCLC others & $2(1.4)$ \\
Grade & \\
G1 & $16(11.5)$ \\
G2 & $57(41.0)$ \\
G3 & $47(33.8)$ \\
Unknown & $19(13.7)$ \\
Surgical margin & \\
Complete & $113(81.3)$ \\
Macroscopic positive & $11(7.9)$ \\
Unknown & $15(10.8)$ \\
\hline
\end{tabular}

SCC, squamous cell carcinoma; NSCLC, non-small cell lung cancer.

vs. 1.043 years, $95 \% \mathrm{CI}, 0.000-3.505, \mathrm{P}=0.615$ ) (Figure $2 A, B$ ). TIM-3 status on TILs could help to predict the RFS and OS of patients simultaneously. High level of TIM-3 on TILs indicated shorter RFS and OS (RFS 1.800 years, 95\% CI, $1.230-2.370$ vs. 0.870 years, $95 \%$ CI, $0.212-1.528, \mathrm{P}=0.048$ ) (OS 2.960 years, $95 \%$ CI, 2.268-3.652 vs. 1.080 years, $95 \%$ CI, 0.228-1.932, $\mathrm{P}=0.034$ ) (Figure 2C,D).

We then separately analyze the prognostic value of TIM3 on both sides in stage I-II and stage III-IV patients. Regretfully, the sample size of tumor TIM3 positive was too small to show any positive results (4 at stage I-II; 5 at stage III-IV). However, we did find that the prognostic value of TIM-3 on TILs was more significant in in stage III patients for both RFS and OS (stage I-II: mRFS $3.210 \mathrm{vs.}$ 1.080 years, $\mathrm{P}=0.008$; mOS 3.440 vs. 1.080 years, $\mathrm{P}=0.001$, Figure $2 E, F$ ) (stage III-IV: mRFS 0.660 vs. 0.607 years, $\mathrm{P}=0.909 ;$ mOS 0.730 vs. 1.060 years, $\mathrm{P}=0.448$ ).

\section{Cox regression for survival analysis}

We computed the HRs of clinical parameters and checkpoint expression levels using univariate and multivariate cox regression. However, the cox regressions showed no correlation with TIM-3 expression on TILs or TCs. Only cancer stage was related with both RFS ( $\mathrm{P}=0.009$, HR 2.543, 95\% CI, 1.265-5.112) and OS ( $\mathrm{P}=0.001$, HR 3.393, 95\% CI, 1.686-6.829) (Tables 4,5). But in stage I-II patients, TIM-3 on TILs showed great prognostic value for 

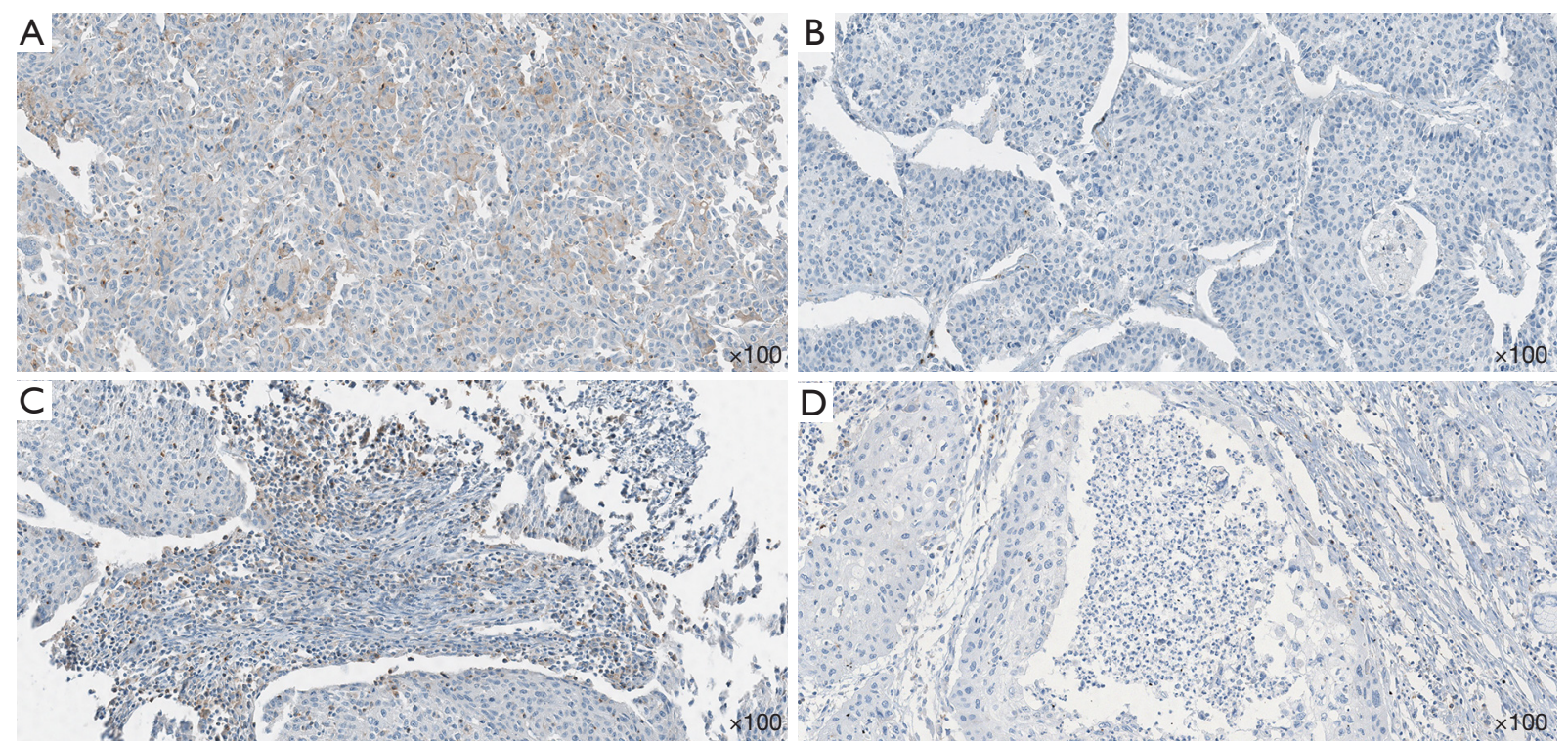

Figure 1 Expression of TIM-3 on tumor cells and TILs. (A) Positive staining of TIM-3 on tumor cells. (B) Negative staining of TIM-3 on tumor cells. (C) Positive staining of TIM-3 on TILs. (D) Negative staining of TIM-3 on TILs. TIM-3, T cell immunoglobulin and mucindomain containing-3; TIL, tumor infiltrating lymphocyte.

OS in multivariate cox regression ( $\mathrm{P}=0.002$, HR $3.912,95 \%$ CI, 1.646-9.296) (Tables 6,7).

\section{Discussion}

The aim of this research was to evaluate TIM-3 expression status on tumor cells and TILs in NSCLC tumor tissue. This study that underlined that TIM-3 expression level on TILs was associated with the expression of other immune checkpoints and the survival time of NSCLC patients. We found TIM-3 was expressed on tumor cells and lymphocytes in both adenocarcinoma and squamous carcinoma. More importantly, high level of TIM-3 on lymphocytes was correlated with early relapse and shorter survival time on NSCLC patients.

TIM-3 is an immune checkpoint protein which can suppress $\mathrm{T}$ cell functions. TIM- 3 was first discovered to screen for $\mathrm{T}$ helper type 1 cells producing IFN- $\gamma(8)$. In a previous study, researchers found that TIM-3 blockade therapy could reverse peripheral tolerance in Th1 cells (19). While in TIM-3-knocked out mouse models, high level of antigen could not induce antigen tolerance (19). The study of TIM-3 was extended to tumor immunity. In melanoma patients, TIM-3 marked a group of CD8(+) lymphocyte that display decreased cytokine production (20). When nonHodgkin lymphoma patients administered with IL-2, TIM-
3 was upregulated and contributed to the development of $\mathrm{T}$ cell exhaustion (21). Several former studies also showed that TIM-3 had similar function like PD-1 and PD-L1 in the impairment of antitumor immunity. TIM3 was detected on several kind of tumor cells including melanoma, gastric cancer, cervical cancer and lung cancer (20,22-24). Published data indicated that TIM-3 upregulation on cancer cells had significant correlation with shorter survival in NSCLC patients (22). However, given the fact that TIM-3 mainly serves its immune suppressive function on T cells, to describe the common TIM-3 status on NSCLC TILs is certainly necessary.

Our study indicated that TIM-3 was widely existed in NSCLC tissue, expressed by both tumor cells and TILs. There was no correlation between TIM-3 expression and clinical features as most immune checkpoint did. However, we discovered a co-expression pattern of TIM-3, PD-1 and PD-L1 on TILs. Meanwhile, although TIM-3 level was much higher on TILs than on tumor cells, patients had TIM-3 positive cancer cells were more likely to have TIM3 positive lymphocytes. This finding was consistent with a recent study using single-cell analysis (25), suggesting high credibility of these results. The co-expression of TIM3, PD-1 and PD-L1 could lead to various consequences. According to previous study, the co-expression pattern may contribute to the development of PD-1(+) TIM-3(+) 
Table 2 Relationship between TIM-3 and clinical data (samples from Medical University of Gdansk)

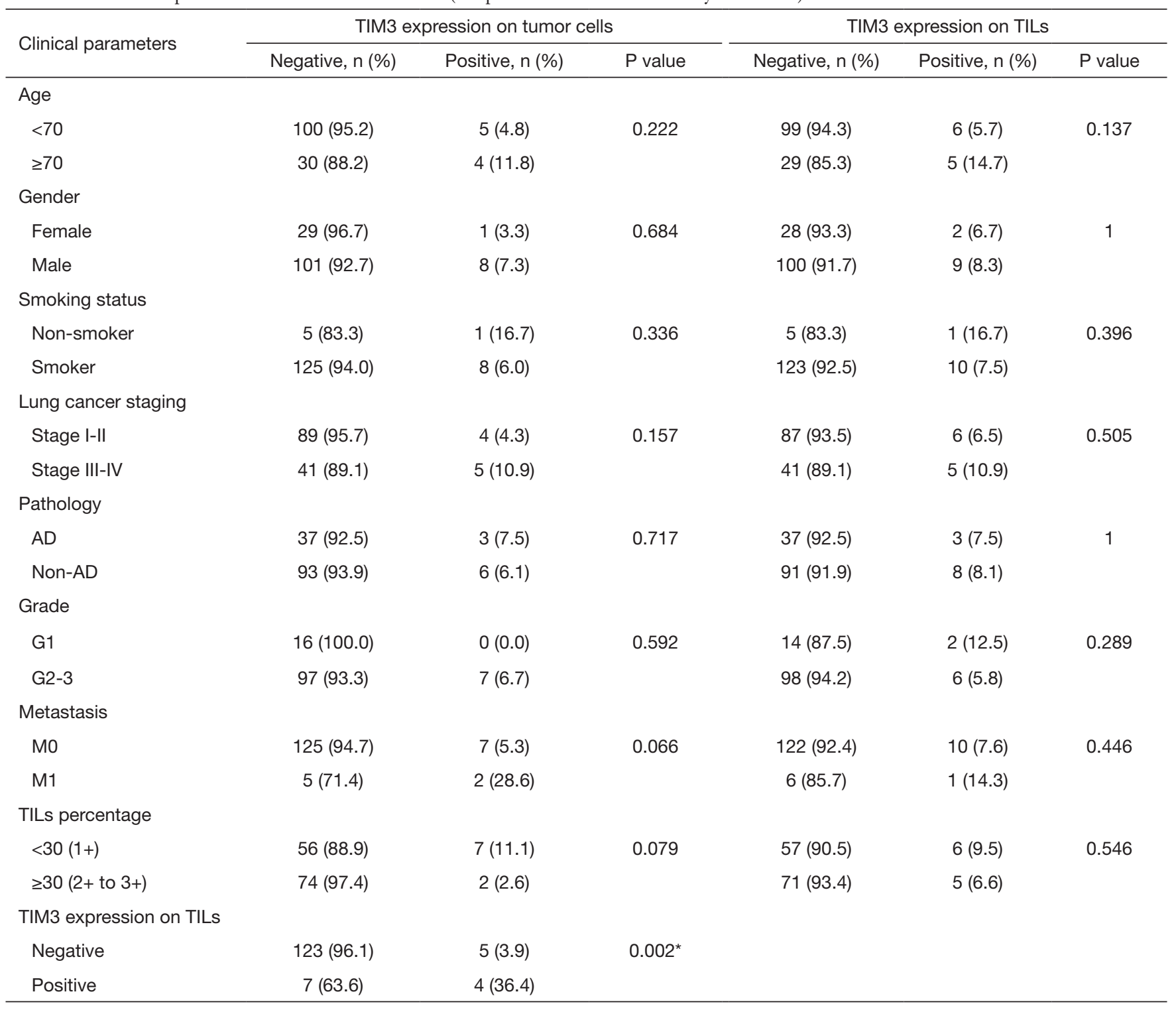

*, $\mathrm{P}<0.05$ indicates statistical significance. AD, Alzheimer's disease; TIL, tumor infiltrating lymphocyte.

Table 3 Relationship between expression level of TIM-3 and other checkpoints

\begin{tabular}{lcccc}
\hline \multirow{2}{*}{ Checkpoint/parameter } & \multicolumn{2}{c}{ TIM-3 on tumor cells } & \multicolumn{2}{c}{ TIM-3 on TILs } \\
\cline { 2 - 4 } \cline { 3 - 4 } PD-L1 on tumor cells & Correlation coefficient & P value & & Correlation coefficient \\
PD-1 on TILs & 0.170 & $0.045^{*}$ & 0.255 & $0.002^{*}$ \\
PD-L1 on TILs & -0.071 & 0.409 & 0.346 & $0 *$ \\
TIM-3 on tumor cells & -0.044 & 0.608 & 0.313 & 0.262 \\
TIL & - & - & $0.002^{*}$ & 0.172 \\
\hline
\end{tabular}

${ }^{*}, \mathrm{P}<0.05$ indicates statistical significance. TIL, tumor infiltrating lymphocyte. 

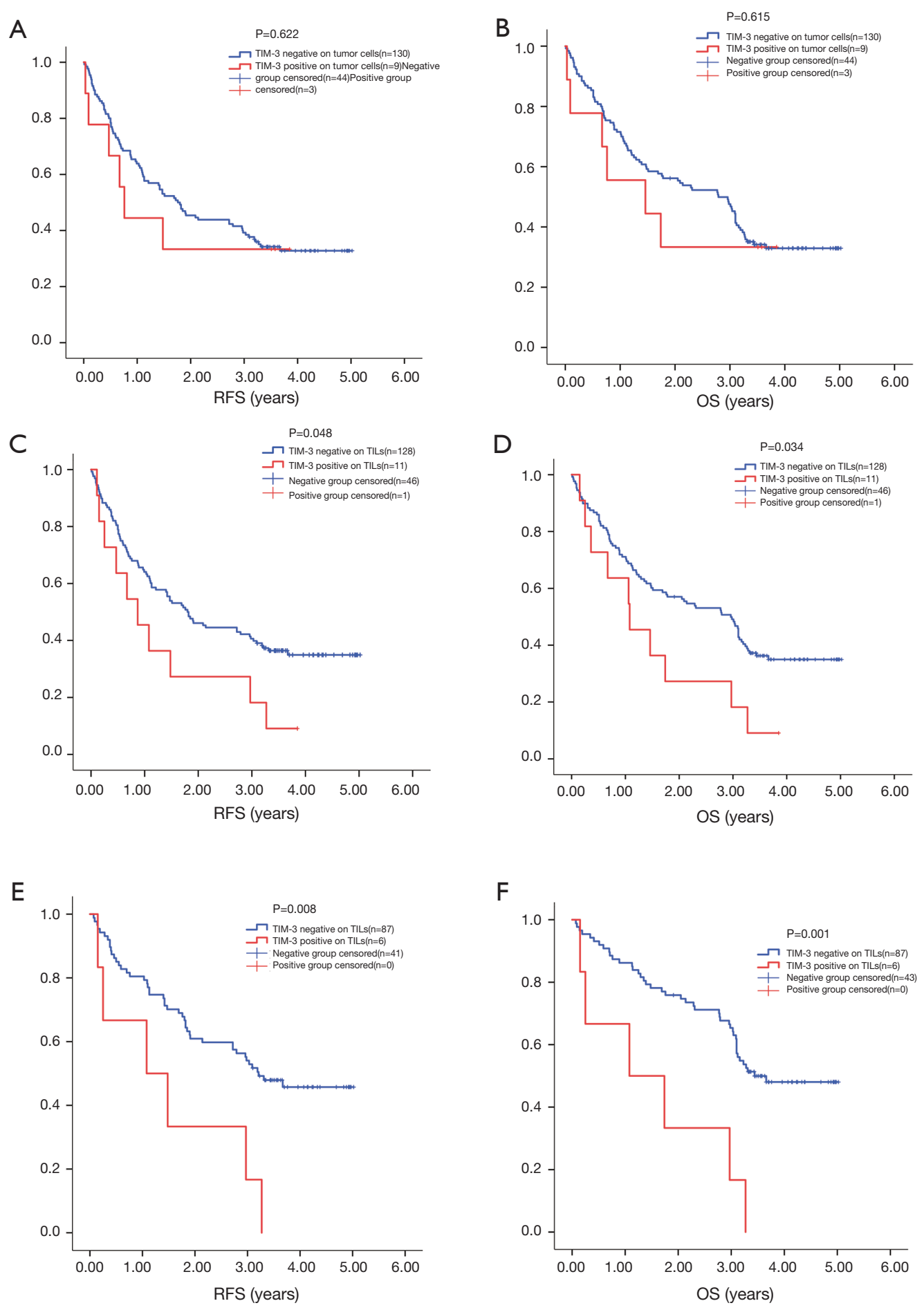

Figure 2 Survival analysis by TIM-3 level on TILs and tumor cells. High level of TIM-3 on TILs indicated shorter RFS (C: RFS 1.800 years, 95\% CI, 1.230-2.370 vs. 0.870 years, 95\% CI, 0.212-1.528, P=0.048) and OS (D: OS 2.960 years, $95 \%$ CI, $2.268-3.652$ vs. 1.080 years, $95 \%$ CI, 0.228-1.932, $\mathrm{P}=0.034)$. TIM-3 level on tumor cells showed no correlation with survival (A: RFS 1.760 years, 95\% CI, 1.246-2.274 vs. 0.760 years, 95\% CI, 0.497-1.023, P=0.622) (B: OS 2.790 years, 95\% CI, 1.970-3.610 vs. 1.043 years, 95\% CI, 0.000-3.505, P=0.615). In stage I-II patients: E: RFS 3.210 vs. 1.080 years, $\mathrm{P}=0.008$; F: OS 3.440 vs. 1.080 years, $\mathrm{P}=0.001$. 
Table 4 COX regression analysis of RFS

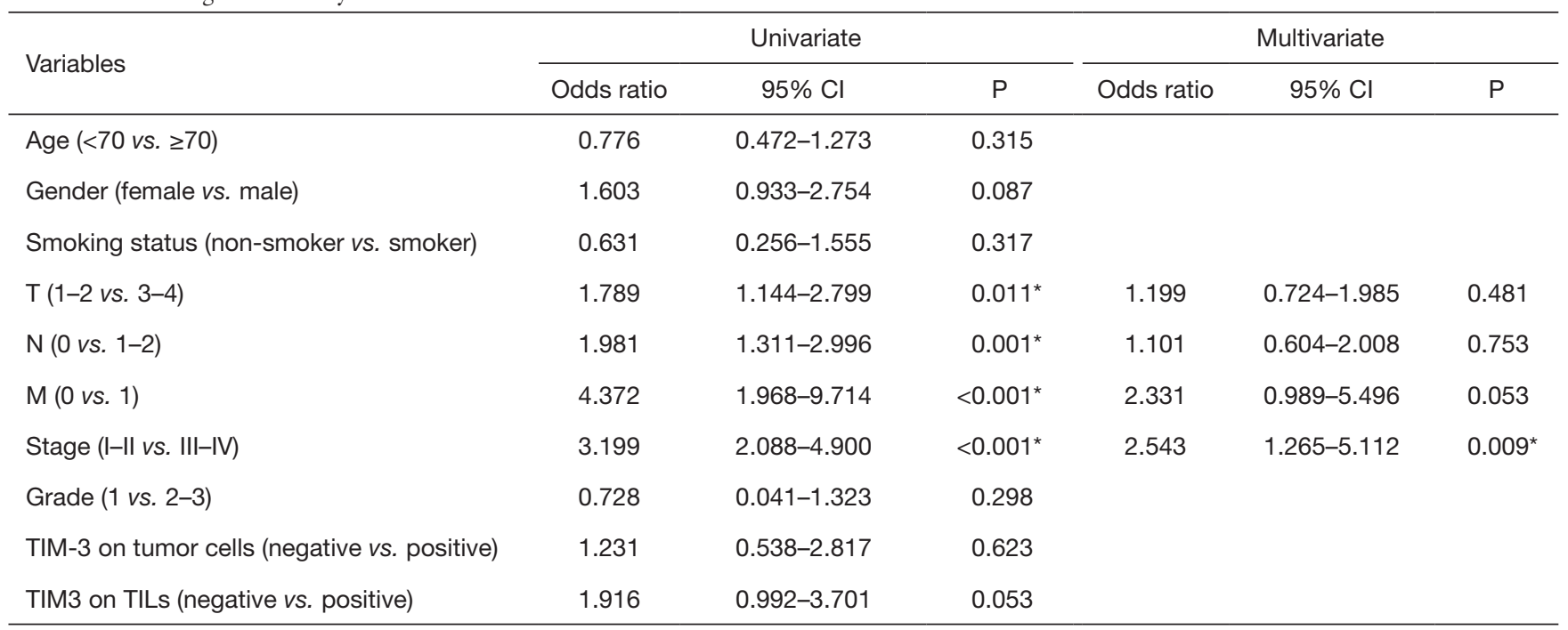

${ }^{*}, \mathrm{P}<0.05$ indicates statistical significance. RFS, recurrence-free survival; TIL, tumor infiltrating lymphocyte.

Table 5 COX regression analysis of OS

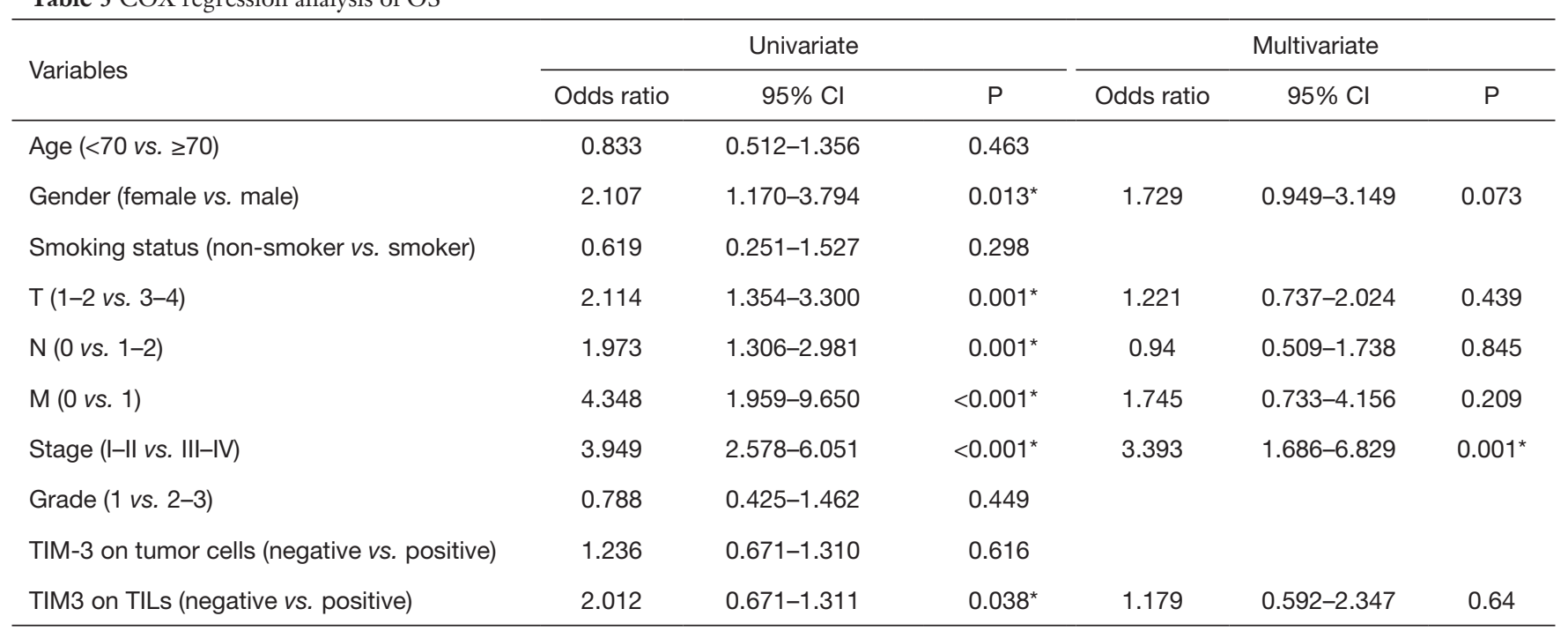

* $\mathrm{P}<0.05$ indicates statistical significance. OS, overall survival; TIL, tumor infiltrating lymphocyte.

CD8(+) phenotype lymphocytes, which was considered the most exhausted T-effector cell phenotype (13). In ovarian cancer, these triple-positive $\mathrm{T}$ cells were correlated with poor prognosis (26). More importantly, TIM-3 could cause adaptive resistance to checkpoint blockade therapy (14). A remarkable work by Datar et al. (25) used multiplexed quantitative immunofluorescence to test PD-1, TIM-3 and anti-lymphocyte activation gene 3 (LAG3, CD223) on two large lung cancer cohort. The correlation between
PD-1 and TIM-3 was found in their study, as well as the prognostic value of TIM-3, which was similar to our results. Furthermore, they detected the co-expression of PD-1, TIM-3 and LAG-3 was related with makers indicating T-cell activation, proliferation and effect function, but also apoptotic markers. Additionally, a former research also showed that a large fraction of TIM-3(+) CD4(+) T cells expressed FOXP3, which is a protein biomarker for immune suppressive regulatory $\mathrm{T}$ cells $(27,28)$. These studies 
Table 6 COX regression analysis of RFS in stage I-II patients

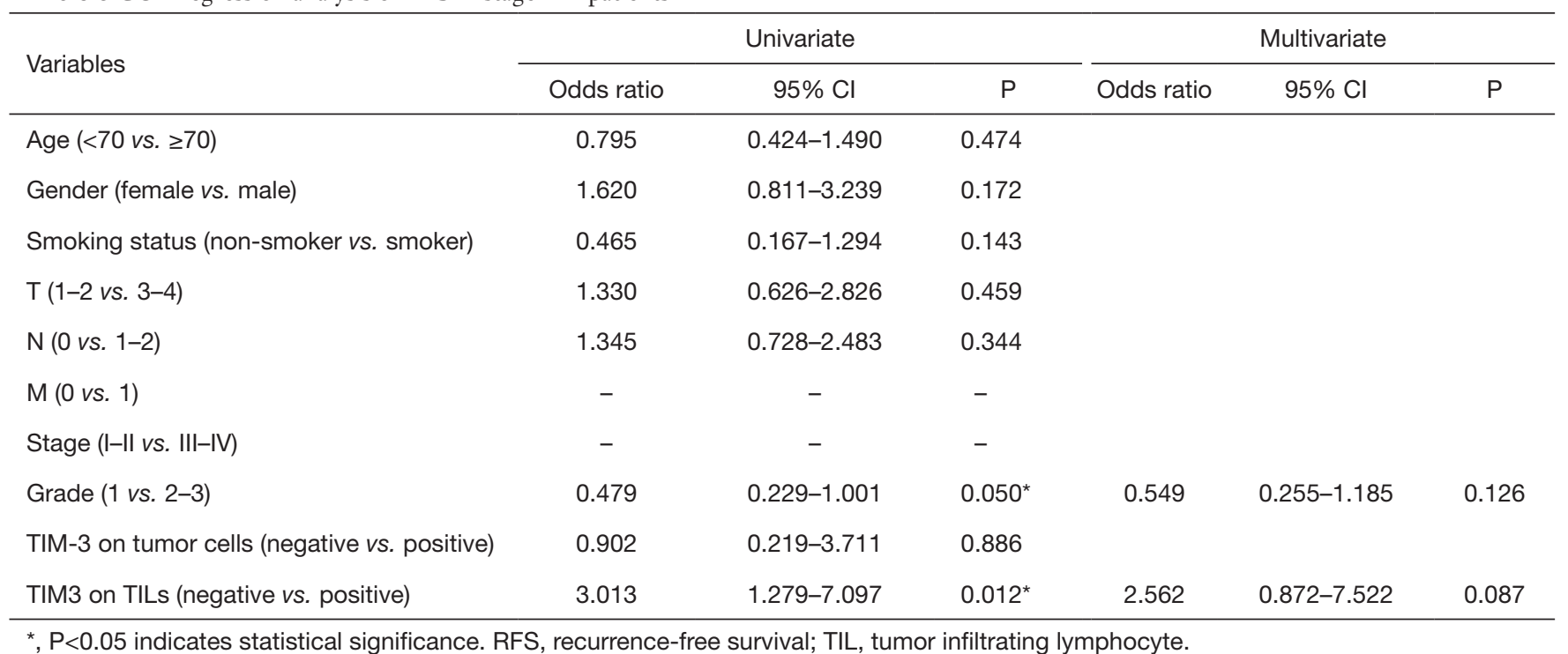

Table 7 COX regression analysis of OS in stage I-II patients

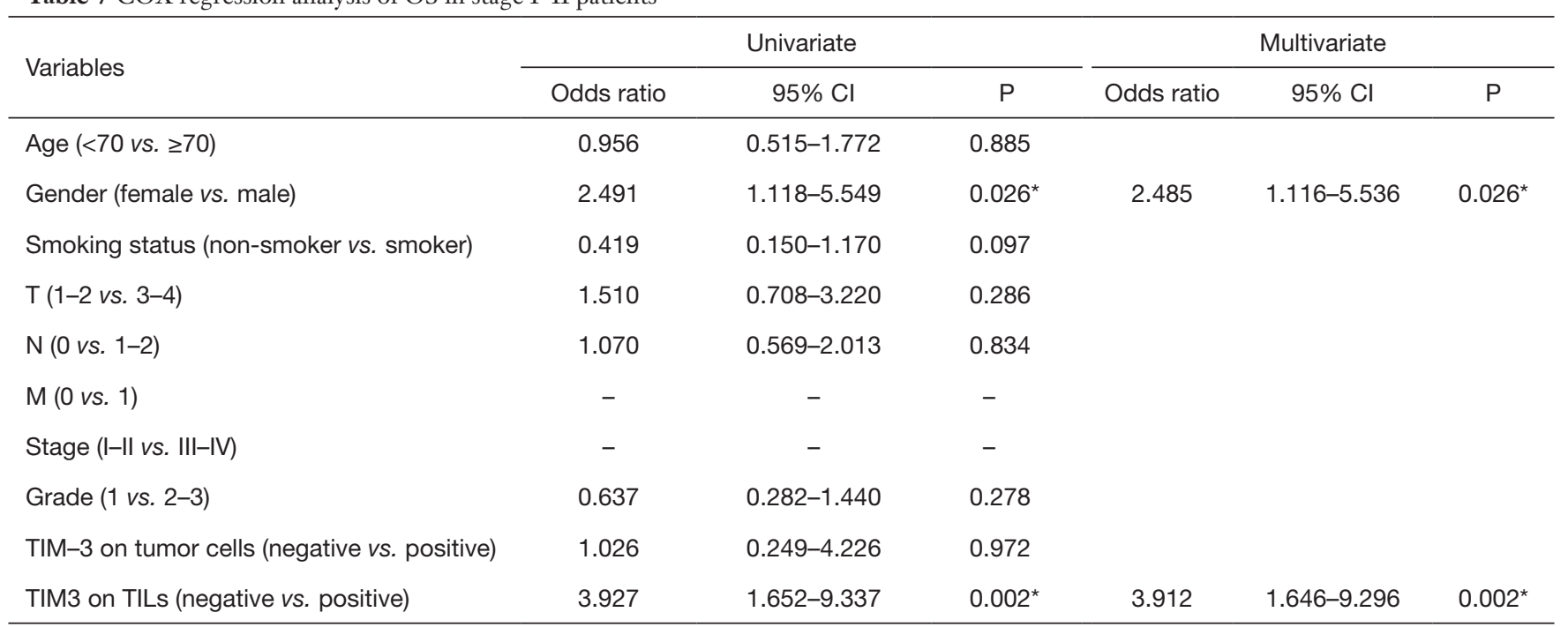

* $\mathrm{P}<0.05$ indicates statistical significance. OS, overall survival; TIL, tumor infiltrating lymphocyte.

together demonstrated that TIM-3 played an important part in T cell immunity dysfunction and might eventually lead to tumor progression. And the upstream signal pathways causing this co-expression of TIM-3 and PD-1/ L1 were also worthy of further investigation. In a recent publication (29) by our group, we analyzed the prognostic value of galectin-9, one ligand of TIM-3, using the same cohort. It was quite intriguing to find that high galectin-9 on tumor side or TILs side could lead to completely diverged outcomes. The association between TIM-3 and galectin-9 expression in situ was also found, suggesting that the main ligand of TIM-3 might be galectin-9 on lymph cells since high galectin-9 on tumor cell led to longer OS.

We also conducted the correlation analysis between TIM-3 and survival. No difference was found when patients who had TIM-3 expression on their cancer cells compared with those whose cancer cells had no TIM3 expression. However, patients had TIM-3 negative 
TILs showed notably longer PFS and OS than those had TIM-3 positive expression on TILs. This finding might support the hypothesis that TIM-3 was more functional on TILs, rather than on tumor cells. A study in mouse models showed that a combined therapy of anti-TIM-3 and anti-PD-L1 antibodies could successfully induce tumor shrinkage (12). An expansion of memory precursor- and effector-like CD8(+) T cells was also detected after this combined therapy, suggesting that the combination of these therapies may have more a profound effect on tumor micro environment (30).

Several clinical trials of anti-TIM3 monoclonal antibodies in patients with advanced solid tumors were currently ongoing. Most of these trials combined TIM-3 blockade with other therapies such as anti-PD1 and antiLAG3 (NCT03311412, NCT02817633). Some added antiTIM-3 to classical regimen with anti-PD-1, chemotherapy and bevacizumab (NCT03307785). LY3321367, an antiTIM-3 monoclonal antibody, has shown been well tolerated as monotherapy or combined with anti-PD-L1 antibody. We suggested that these drugs in trails had a promising prospective when combined or in the treatment of PD-1/L1 antibody resistant tumors.

Limitations of our study are obvious. First, this was an exploratory, hypothesis generating retrospective study with a rather small and heterogeneous cohort. Especially when tumor-side TIM-3 positive patients only accounted for $6.5 \%$ of total, we had to admit that the data of TIM- 3 on tumor cell was insufficient. Second, without a settled cutoff for TIM-3, we ran the survival analysis repeatedly with different cutoffs and decide the optimized cutoffs when the survival difference between groups was maximized. Third, the lack of clinical information like mutation type and treatment data limited the depth of this research. For further investigation, a prospective study with larger cohort is needed and more elaborate experiment design may be expected. What's more, several recent studies found that co-expression of inhibitory receptors could also indicate a group of activated immune cells, suggesting that IHC test with a larger panel may reveal more comprehensive results $(25,31)$.

\section{Conclusions}

In this study, we discovered TIM-3 expression on NSCLC cancer cells and TILs. We also found a synergistic expression pattern of TIM-3, PD-1 and PD-L1 on NSCLC TILs. Furthermore, TIM-3 level on TILs was notably correlated with early postoperative recurrence and shortened survival time. More exploration needs to be conducted to reveal the mechanism behind.

\section{Acknowledgments}

Funding: This study was supported in part by a grant from National Natural Science Foundation of China (81802255), Shanghai Pujiang Program (17PJD036) and a grant from Shanghai Municipal Commission of Health and Family Planning Program (20174Y0131), National key research \& development project (2016YFC0902300), Major disease clinical skills enhancement program of three year action plan for promoting clinical skills and clinical innovation in municipal hospitals, Shanghai Shen Kang Hospital Development Center Clinical Research Plan of SHDC (16CR1001A), "Dream Tutor" Outstanding Young Talents Program (fkyq1901), key disciplines of Shanghai Pulmonary Hospital (2017ZZ02012), grant of Shanghai Science and Technology Commission (16JC1405900).

\section{Footnote}

Conflicts of interest: The authors have no conflicts of interest to declare.

Etbical Statement: The authors are accountable for all aspects of the work in ensuring that questions related to the accuracy or integrity of any part of the work are appropriately investigated and resolved. This study was approved by the committee of Medical University of Gdansk, Poland (ethical number 15-235). Written informed consent was obtained from the patient for publication of this manuscript and any accompanying images.

\section{References}

1. Chen $W$, Zheng $\mathrm{R}$, Zhang $\mathrm{S}$, et al. Lung cancer incidence and mortality in China, 2009. Thorac Cancer 2013;4:102-8.

2. Siegel RL, Miller KD, Jemal A. Cancer statistics, 2019. CA Cancer J Clin 2019;69:7-34.

3. Cameron L, Solomon B. New Treatment Options for ALK-Rearranged Non-Small Cell Lung Cancer. Curr Treat Options Oncol 2015;16:49.

4. Zhou C, Wu YL, Chen G, et al. Final overall survival results from a randomised, phase III study of erlotinib versus chemotherapy as first-line treatment of EGFR mutation-positive advanced non-small-cell lung 
cancer (OPTIMAL, CTONG-0802). Ann Oncol 2015;26:1877-83.

5. Errico A. Melanoma: CheckMate 067--frontline nivolumab improves PFS alone or in combination with ipilimumab. Nat Rev Clin Oncol 2015;12:435.

6. Khandekar MJ, Jain R. Smooth sailing for immunotherapy for unresectable stage III non-small cell lung cancer: the PACIFIC study. Transl Cancer Res 2018;7:S16-20.

7. Larkin J, Minor D, D'Angelo S, et al. Overall Survival in Patients With Advanced Melanoma Who Received Nivolumab Versus Investigator's Choice Chemotherapy in CheckMate 037: A Randomized, Controlled, Open-Label Phase III Trial. J Clin Oncol 2018;36:383-90.

8. Monney L, Sabatos CA, Gaglia JL, et al. Th1-specific cell surface protein Tim-3 regulates macrophage activation and severity of an autoimmune disease. Nature 2002;415:536-41.

9. Morimoto K, Hosomi S, Yamagami H, et al. Dysregulated upregulation of T-cell immunoglobulin and mucin domain-3 on mucosal $\mathrm{T}$ helper 1 cells in patients with Crohn's disease. Scand J Gastroenterol 2011;46:701-9.

10. Wherry EJ, Kurachi M. Molecular and cellular insights into T cell exhaustion. Nat Rev Immunol 2015;15:486-99.

11. Jones RB, Ndhlovu LC, Barbour JD, et al. Tim-3 expression defines a novel population of dysfunctional $\mathrm{T}$ cells with highly elevated frequencies in progressive HIV-1 infection. J Exp Med 2008;205:2763-79.

12. Sakuishi K, Apetoh L, Sullivan JM, et al. Targeting Tim-3 and PD-1 pathways to reverse T cell exhaustion and restore anti-tumor immunity. J Exp Med 2010;207:2187-94.

13. Zhou Q, Munger ME, Veenstra RG, et al. Coexpression of Tim-3 and PD-1 identifies a CD8+ T-cell exhaustion phenotype in mice with disseminated acute myelogenous leukemia. Blood 2011;117:4501-10.

14. Koyama S, Akbay EA, Li YY, et al. Adaptive resistance to therapeutic PD-1 blockade is associated with upregulation of alternative immune checkpoints. Nat Commun 2016;7:10501.

15. He Y, Yu H, Rozeboom L, et al. LAG-3 Protein Expression in Non-Small Cell Lung Cancer and Its Relationship with PD-1/PD-L1 and Tumor-Infiltrating Lymphocytes. J Thorac Oncol 2017;12:814-23.

16. He Y, Rozeboom L, Rivard CJ, et al. MHC class II expression in lung cancer. Lung Cancer 2017;112:75-80.
17. Ghebeh H, Mohammed S, Al-Omair A, et al. The B7-H1 (PD-L1) T lymphocyte-inhibitory molecule is expressed in breast cancer patients with infiltrating ductal carcinoma: correlation with important high-risk prognostic factors. Neoplasia 2006;8:190-8.

18. Schalper KA, Velcheti V, Carvajal D, et al. In situ tumor PD-L1 mRNA expression is associated with increased TILs and better outcome in breast carcinomas. Clin Cancer Res 2014;20:2773-82.

19. Sabatos CA, Chakravarti S, Cha E, et al. Interaction of Tim-3 and Tim-3 ligand regulates T helper type 1 responses and induction of peripheral tolerance. Nat Immunol 2003;4:1102-10.

20. Fourcade J, Sun Z, Benallaoua M, et al. Upregulation of Tim-3 and PD-1 expression is associated with tumor antigen-specific CD8+ T cell dysfunction in melanoma patients. J Exp Med 2010;207:2175-86.

21. Yang ZZ, Grote DM, Ziesmer SC, et al. IL-12 upregulates TIM-3 expression and induces $T$ cell exhaustion in patients with follicular B cell non-Hodgkin lymphoma. J Clin Invest 2012;122:1271-82.

22. Zhuang X, Zhang X, Xia X, et al. Ectopic Expression of TIM-3 in Lung Cancers A Potential Independent Prognostic Factor for Patients With NSCLC. Am J Clin Pathol 2012;137:978-85.

23. Cao Y, Zhou X, Huang X, et al. Tim-3 expression in cervical cancer promotes tumor metastasis. PLoS One 2013;8:e53834.

24. Jiang J, Jin MS, Kong F, et al. Decreased galectin-9 and increased Tim-3 expression are related to poor prognosis in gastric cancer. PLoS One 2013;8:e81799.

25. Datar I, Sanmamed MF, Wang J, et al. Expression Analysis and Significance of PD-1, LAG-3, and TIM-3 in Human Non-Small Cell Lung Cancer Using Spatially Resolved and Multiparametric Single-Cell Analysis. Clin Cancer Res 2019;25:4663-73.

26. Fucikova J, Rakova J, Hensler M, et al. TIM-3 dictates functional orientation of the immune infiltrate in ovarian cancer. Clin Cancer Res 2019;25:4820-31.

27. Hori S, Nomura T, Sakaguchi S. Control of regulatory T cell development by the transcription factor Foxp3. Science 2003;299:1057-61.

28. Gao X, Zhu Y, Li G, et al. TIM-3 expression characterizes regulatory $\mathrm{T}$ cells in tumor tissues and is associated with lung cancer progression. PLoS One 2012;7:e30676.

29. He Y, Jia K, Dziadziuszko R, et al. Galectin-9 in non-small cell lung cancer. Lung Cancer 2019;136:80-5.

30. Kurtulus S, Madi A, Escobar G, et al. Checkpoint 
Blockade Immunotherapy Induces Dynamic Changes in PD-1-CD8+ Tumor-Infiltrating T Cells. Immunity 2019;50:181-94.e6.

31. Xiong H, Mittman S, Rodriguez R, et al.
Coexpression of Inhibitory Receptors Enriches for Activated and Functional CD8+ T Cells in Murine Syngeneic Tumor Models. Cancer Immunol Res 2019;7:963-76.

Cite this article as: Jia K, He Y, Dziadziuszko R, Zhao S, Zhang X, Deng J, Wang H, Hirsch FR, Zhou C, Yu H, Zhang L. $\mathrm{T}$ cell immunoglobulin and mucin-domain containing-3 in nonsmall cell lung cancer. Transl Lung Cancer Res 2019;8(6):895906. doi: 10.21037/tlcr.2019.11.17 
Supplementary

Table S1 Univariate and multivariate analysis for prediction of TIM-3 expression on tumor cells in NSCLC patients

\begin{tabular}{|c|c|c|c|c|c|c|}
\hline \multirow{2}{*}{ Variables } & \multicolumn{3}{|c|}{ Univariate } & \multicolumn{3}{|c|}{ Multivariate } \\
\hline & Odds ratio & $95 \% \mathrm{Cl}$ & $P$ & Odds ratio & $95 \% \mathrm{Cl}$ & $\mathrm{P}$ \\
\hline Age (<70 y vs. $\geq 70$ y) & 2.667 & $0.673-10.564$ & 0.163 & & & \\
\hline Sex (female vs. male) & 2.297 & $0.276-19.127$ & 0.442 & & & \\
\hline Smoking status (nonsmoker vs. smoker) & 0.32 & $0.033-3.075$ & 0.324 & & & \\
\hline Pathological type (non-adenocarcinoma vs. adenocarcinoma) & 1.257 & $0.299-5.290$ & 0.755 & & & \\
\hline PD-L1 on tumor cells (negative vs. positive) & 5.318 & $1.298-21.785$ & $0.02^{\star}$ & 5.318 & $1.298-21.785$ & $0.02^{*}$ \\
\hline PD-1 on TILs (negative vs. positive) & 0.64 & $0.153-2.672$ & 0.541 & & & \\
\hline PD-L1 on TILs (negative vs. positive) & 0.488 & $0.097-2.455$ & 0.383 & & & \\
\hline
\end{tabular}

*, $\mathrm{P}<0.05$ indicates statistical significance. NSCLC, non-small cell lung cancer; TIL, tumor infiltrating lymphocyte.

Table S2 Univariate and multivariate analysis for prediction of TIM-3 expression on TILs in NSCLC patients

\begin{tabular}{|c|c|c|c|c|c|c|}
\hline \multirow{2}{*}{ Variables } & \multicolumn{3}{|c|}{ Univariate } & \multicolumn{3}{|c|}{ Multivariate } \\
\hline & Odds ratio & $95 \% \mathrm{Cl}$ & $\mathrm{P}$ & Odds ratio & $95 \% \mathrm{Cl}$ & $\mathrm{P}$ \\
\hline Age (<70 y vs. $\geq 70$ y) & 2.845 & $0.809-9.998$ & 0.103 & & & \\
\hline Sex (female vs. male) & 1.26 & $0.257-6.169$ & 0.776 & & & \\
\hline Smoking status (nonsmoker vs. smoker) & 0.407 & $0.043-3.825$ & 0.431 & & & \\
\hline Pathological type (non-adenocarcinoma vs. adenocarcinoma) & 0.922 & $0.232-3.669$ & 0.909 & & & \\
\hline Stage (I-II vs. III-IV) & 1.768 & $0.510-6.132$ & 0.369 & & & \\
\hline PD-L1 on tumor cells (negative vs. positive) & 1.275 & $0.255-6.364$ & 0.767 & & & \\
\hline PD-1 on TILs (negative vs. positive) & 1.644 & $0.477-5.669$ & 0.431 & & & \\
\hline PD-L1 on TILs (negative vs. positive) & 2.291 & $0.662-7.929$ & 0.191 & & & \\
\hline
\end{tabular}

NSCLC, non-small cell lung cancer; TIL, tumor infiltrating lymphocyte. 